\title{
Exercícios físicos e redução de quedas em idosos: uma revisão sistemática
}

\author{
Physical exercises and risk of fall reduction in elderly: a systematic \\ review
}
Paulo Cesar Barauce Bento ${ }^{1}$ André Luíz Félix Rodacki 2 Diogo Homann Neiva Leite ${ }^{2}$

1. Universidade Federal do Paraná. Programa de Pós-Graduação em Educação Física. Departamento de Educação Física. Curitiba, PR. Brasil.

Recebido em 24/06/10 Revisado em 12/07/10 Aprovado em 04/08/10
Resumo - As quedas estão associadas à morbidade e mortalidade em idosos. O objetivo dessa revisão sistemática foi identificar, descrever e analisar o efeito do exercício físico na redução do risco de quedas em idosos. Realizou-se busca no site do Centro Latino-Americano de Informação em Ciências da Saúde (BIREME), bases de dados Medline/Pubmed, Lilacs e SciELO, nos anos de 1999-2009, com os seguintes descritores em inglês: "acidental falls", "eldery", "exercise“. Foram encontrados 385 artigos e, após a aplicação dos critérios de inclusão e exclusão, selecionaram-se 10 artigos que fizeram parte da análise final. Todos os artigos incluídos avaliaram indivíduos a partir de 60 anos de idade, submetidos a programas de exercícios físicos. Os resultados sugerem que o exercício físico, de forma isolada, é capaz de reduzir o risco de quedas, isto ficou evidente em sete dos estudos avaliados. Dentre os principais componentes de cada programa, houve predominância entre treinamento de força e equilíbrio, além de exercicios de coordenação, flexibilidade e aeróbios. Entretanto, não há indicação clara em relação a frequência, duração e intensidade das sessões. Conclui-se que estudos que associaram componentes de força e/ou equilíbrio, além de outras formas de intervenção, realizados, no mínimo, duas vezes por semana e que tenham acompanhado os indivíduos, em média, de 3 a 6 meses após a intervenção, mostraram-se mais efetivos em reduzir e prevenir as quedas em idosos vivendo na comunidade. No entanto, é necessário que os estudos apresentem com mais clareza alguns aspectos metodológicos que permitam a sua reprodução e comparação dos seus resultados.

Palavras-chave: Quedas; Idoso; Exercício.

Abstract - Falls are associated with morbidity and mortality in older adults. The aim of this systematic review was to identify, describe and analyze the effects of physical exercise programs on the reduction of the risk of falls in adults above the age of 60. For this purpose, the Medline/ Pubmed, Lilacs, and SciELO databases available at the site of the Latin American Center of Information in Health Sciences (BIREME) were searched for articles comprising the period between 1999 and 2009 using the following keywords: accidental falls, elderly, exercise. A total of 385 publications were identified and 10 articles that met the criteria established in this study were selected. Strength and balance activities were the most frequent components of the exercise programs, in addition to coordination, flexibility and aerobic exercise. However, there was no clear information regarding the frequency, duration and intensity of the sessions. It was concluded that programs combining strength and balance components with other interventions, performed at least twice a week, and monitoring the participants for 3 to 6 months after intervention were the most effective in reducing and preventing falls in older adults. However, a more detailed presentation of some methodological aspects is necessary to permit the reproduction of these studies and the comparison of their results.

Key words: Falls; Elderly; Exercise. 


\section{INTRODUÇÃO}

As quedas acidentais estão entre os principais problemas de saúde que afetam as pessoas a partir dos sessenta anos de idade e estão associadas à morbidade, redução da funcionalidade perda da independência e, em alguns casos, em morte. Além desses problemas, existe, ainda, elevado custo financeiro e social ${ }^{1}$. O risco aumenta a partir dos sessenta anos, sendo que aos sessenta e cinco anos ou mais pode atingir entre 35\% a 40\% das pessoas nos Estados Unidos ${ }^{2}$.

Os fatores de risco associados às quedas podem ser classificados em extrínsecos e intrínsecos. Dentre os fatores extrínsecos pode-se destacar a qualidade e intensidade da iluminação, superfícies irregulares, tapetes soltos, condições do piso, uso combinado de medicações e os riscos associados às próprias atividades que o idoso está realizando. Dentre os fatores intrínsecos, destacam-se a redução da força muscular, alterações de equilíbrio, modificações no padrão da marcha, déficit visual, perdas funcionais e cognitivas ${ }^{1-5}$.

A possibilidade de ocorrência de quedas pode aumentar significativamente e varia entre $27 \%$ quando em presença de um fator de risco e $78 \%$ para quatro ou mais fatores associados. Como consequência, as quedas podem ocasionar sérias lesões, dentre as quais a mais frequente é a fratura. As fraturas afetam de $10 \%$ a $15 \%$ dos idosos que caem. Dentre os idosos que experimentam quedas, $75 \%$ não se recuperam totalmente e têmm redução em sua capacidade funcional ${ }^{6}$.

A atividade física regular é importante para a manutenção da funcionalidade, mas quando o idoso tem uma queda há diminuição da autoconfiança, o que pode levar a menor nível de atividade física, ocasionando redução da força muscular e consequentemente, um aumento do risco de quedas ${ }^{6}$. O objetivo desta revisão foi sistematizar os achados em relação aos efeitos dos diferentes programas de exercícios na redução do risco de quedas em idosos e avaliar as evidências de tais resultados.

\section{PROCEDIMENTOS METODOLÓGICOS}

A busca de artigos foi realizada de forma sistemática através do site do Centro Latino-Americano de Informação em Ciências da Saúde (BIREME) que engloba as bases de dados Medline/Pubmed (National Library of Medicine), Lilacs (Literatura Latino-americana e do Caribe de Informação em Ciências da Saúde) e SciELO (Scientific Electronic
Library Online). Os seguintes descritores foram utilizados em associação, estando os mesmos presentes como descritores em ciências da saúde (DeCS): "accidental falls", "elderly" e "exercise".

A associação dos 3 descritores gerou 730 resultados,totalizando em 729 artigos. Destes, 545 estavam relacionados diretamente a idosos, dos quais, 466 artigos estavam em língua inglesa. Por fim, foram considerados apenas os artigos publicados na última década (1999-2009), que totalizaram 385 estudos. Após tais procedimentos, foi realizada uma busca de forma independente por dois autores do manuscrito que aplicaram os critérios de inclusão e exclusão descritos no quadro 1 .

Quadro 1. Critérios de inclusão e exclusão empregados para a seleção de estudos.

\begin{tabular}{|l|}
\hline Critérios de inclusão \\
\hline Idosos não-institucionalizados \\
Idosos vivendo de forma independente na sociedade \\
Idosos que não estivessem ativos pelo menos 3 meses \\
antes de serem feitos os estudos iniciais \\
Estudos destinados a avaliar a modificação do número de \\
quedas durante e/ou após a intervenção \\
Exercícios realizados em grupo com um programa estrutu- \\
rado e regular \\
Artigos originais \\
\hline Critérios de exclusão \\
\hline Estudos realizados com grupos em condições especiais de \\
saúde \\
Estudos que avaliaram apenas as modificações nos fatores \\
de risco de quedas \\
Estudos que avaliaram modificações do medo em relação \\
às quedas \\
Artigos de revisão, cartas aos editores, protocolos de estu- \\
do, comentários, teses, dissertações \\
Programas de intervenção com solicitação/incentivo aos \\
indivíduos realizarem exercícios em casa \\
Programas de intervenção associado à suplementação ou \\
medicação
\end{tabular}

Os estudos incluídos na revisão foram analisados quanto a sua qualidade metodológica, segundo um protocolo de avaliação criado para este estudo, composto por 11 critérios que os autores julgaram importantes para quantificação da qualidade, os quais foram considerados na discussão dos resultados de cada artigo. A pontuação de qualidade também foi realizada por dois investigadores de forma independente e dúvidas surgidas foram analisadas em reunião de consenso entre os mesmos. Quanto maior a pontuação melhor a qualidade do artigo. Os primeiros quatro componentes analisaram a existência de grupo controle, alocação aleatória de sujeitos nos grupos experimentais, similaridade entre 
as características dos grupos antes da intervenção e cálculo amostral. O quinto componente considerou o acompanhamento (follow-up), após o término da intervenção, enquanto o sexto componente observou se o programa de intervenção considerou apenas a aplicação de exercícios físicos sem associação com outros recursos (estratégias relacionadas ao melhoramento da visão, estratégias e/ou aconselhamentos relacionados a melhoramento de cuidados a serem tomados em casa em relação aos fatores de risco a quedas). O sétimo componente analisou se o programa de exercícios era estruturado e supervisionado e com frequência semanal de, no mínimo, duas vezes semanais (considerado regular). O nono componente detectou o emprego de instrumentos confiáveis para a avaliação, considerando instrumentos referenciados no corpo do texto dos artigos ou que são internacionalmente conhecidos. Os aspectos metodológicos podem ser observados na tabela 1 .

\section{RESULTADOS}

A partir dos 385 artigos selecionados e após a verificação dos critérios propostos, foram incluídos 10 artigos para análise na revisão sistemática (Figura 1).

\section{Componentes dos exercicios físicos}

Foram encontradas modalidades de exercícios que compreenderam: ciclismo $^{7}$, caminhada ${ }^{7-9}$, Tai $\mathrm{Chi}^{10,11}$, dança ${ }^{10}$ e procedimentos terapêuticos ${ }^{12,13}$. Alguns programas alternativos foram propostos, os quais envolveram exercícios que buscavam simular atividades da vida diária ${ }^{9}$, além de um programa denominado "workstation", um circuito de exercícios com atividades cotidianas e estímulos específicos para desafiar o equilíbrio ${ }^{14}$.

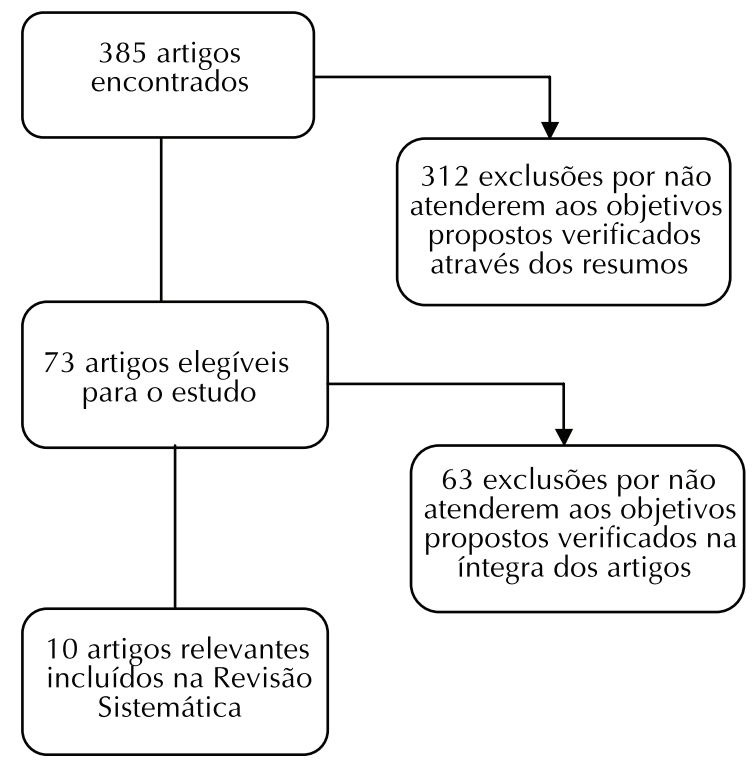

Figura 1. Processo de seleção dos estudos incluídos na revisão.

Dentre os principais componentes de cada programa, houve predominância entre treinamento de força e equilíbrio, além de exercícios de coordenação, flexibilidade e aeróbios. Dos 10 estudos incluídos nesta revisão, sete continham exercícos que visavam a melhoria da capcaidade dos sujeitos em desenvolver força em suas rotinas de treinamento, sendo que os mesmos utilizaram como forma de sobrecarga o próprio peso corporal ${ }^{13-15}$, elásticos ${ }^{7,15}$ e pesos ou implementos na sua execução ${ }^{7,10,16,18}$. A intensidade proposta foi relativa à carga máxima observada em teste de 1 repetição máxima (1RM) em apenas um estudo ${ }^{10}$. Nos demais estudos, a

Tabela 1. Qualidade metodológica dos estudos analisados

\begin{tabular}{|c|c|c|c|c|c|c|c|c|c|c|c|c|c|}
\hline & Autor & 1 & 2 & 3 & 4 & 5 & 6 & 7 & 8 & 9 & 10 & 11 & Total \\
\hline 2001 & Hauer et al. ${ }^{(10)}$ & + & + & + & + & - & + & + & + & + & + & + & 10 \\
\hline 2003 & Steadman et al. (12) & - & + & + & + & - & - & + & - & + & + & - & 6 \\
\hline 2004 & Ballard et al. (15) & + & + & + & + & + & - & + & + & + & + & - & 9 \\
\hline 2004 & Nitz \& Choy ${ }^{(14)}$ & - & + & + & - & - & - & - & - & + & + & + & 5 \\
\hline 2005 & Li et al. ${ }^{(11)}$ & + & + & + & - & - & + & + & + & + & + & + & 9 \\
\hline 2005 & Means et al. ${ }^{(8)}$ & + & + & + & + & - & + & + & - & + & + & + & 9 \\
\hline 2006 & Weerdesteyn et al. (9) & + & - & + & - & - & - & + & - & + & + & + & 6 \\
\hline
\end{tabular}


intensidade foi relatada em termos absolutos ${ }^{7}$, com base na percepção subjetiva de esforço ${ }^{8}$, onde apenas o número de séries e repetições ${ }^{15}$ foi indicado. Em alguns casos, apenas houve relato de que a intensidade foi progressiva ${ }^{9,11-14,16 .}$

O treinamento voltado para o equilíbrio, mesmo que não tenha sido o principal componente, foi a forma utilizada em todos os estudos incluídos nesta revisão. $\bigcirc$ treinamento do equilíbrio foi associado ao treinamento de força em sete estudos $7,10,13$. 15 e com programa de fisioterapia convencional ${ }^{12,13}$, também incluiram exercicios de equilíbrio que foram asociados a diferenciados tipos de exercícios (alongamento, força, aeróbicos e de coordenação motora). O Tai Chi, como única forma de exercício, também foi utilizado ${ }^{11}$. O treinamento de equilíbrio sem estar associado a outro tipo de intervenção (força, flexibilidade, resistência), foi desenvolvido em apenas dois estudos ${ }^{12,9}$.

Foram aplicados nos estudos exercícios de equilíbrio estático ${ }^{10,13,15}$, equilíbrio dinâmico ${ }^{7,9,11}$ enquanto outros ${ }^{8,12,14,16}$ combinaram as duas formas de intervenção.

O tempo de duração dos programas variou entre cinco semanas ${ }^{9}$ e um ano ${ }^{16}$. Foram encontrados estudos com duração de seis ${ }^{8,12}$, oito ${ }^{13}$, dez $^{14}$, doze $^{7,10}$, quinze ${ }^{15}$ e vinte e seis semanas ${ }^{11}$. Em relação à frequência semanal, apenas um estudo ${ }^{14}$ realizou intervenção uma única vez na semana. A maioria dos estudos foi realizada com uma frequência de três sessões semanais 7,8,10,11,13,15,16 com exceção de dois estudos em que os exercícios eram realizados duas vezes semanais ${ }^{9,12}$.

\section{Período do acompanhamento (follow-up) e} forma de avaliação da ocorrência de quedas O período de acompanhamento (folow-up) foi de seis $^{9,11}$, dez $^{13}$ e doze meses ${ }^{15}$. Períodos mais curtos de acompanhamento foram observados, sendo de dezoito $^{12}$ e doze semanas ${ }^{10,14}$. Dos 10 estudos analisados, dois deles não fizeram acompanhamento após o período de intervenção proposto ${ }^{7,16}$.

As avaliações sobre a ocorrência de quedas foram realizadas no período de seguimento, mediante anotações dos participantes em diários, enviados normalmente por correio ou telefone, com periodicidade mensal ${ }^{9,11,14,16}$ ou quinzenal ${ }^{7,10,13}$. Steadman et al. ${ }^{12}$, avaliaram as quedas de forma retrospectiva, durante o mês que antecedeu a intervenção, ao final e após 6, 12 e 18 semanas. Ballard et al. ${ }^{15}$, apesar de ter realizado 6 avaliações referentes às quedas durante 15 semanas de intervenção, realizou somente 1 avaliação (1 ano) após o término do programa.,
A forma de análise em relação à ocorrência de quedas, durante ou após o período de exercício, variou entre os diferentes estudos. Steadman et al. ${ }^{12}$, procurou descrever as quedas ocorridas no mês anterior a cada um dos 4 momentos de avaliação propostos. Nitz \& $\mathrm{Choy}^{14}$, buscaram comparar a quantidade de quedas referentes ao ano anterior ao início do estudo com as quedas ocorridas durante a intervenção e durante o período de acompanhamento. Means et al. ${ }^{8}$ e Weerdesteyn et al. ${ }^{9}$, compararam o número de quedas ocorridas 6 meses antes do início da intervenção com aquelas ocorridas 6 meses após o término da intervenção que teve uma duração de apenas 6 semanas. Neste segundo estudo, os autores avaliaram as quedas de forma prospectiva, a fim de tentar garantir maior fidedignidade aos resultados. Ballard et al. ${ }^{15}$, relataram o número de quedas ocorridas durante o período de intervenção e após o período de acompanhamento, enquanto Rubenstein et al. ${ }^{7}$ e Shumway-Cook et al. ${ }^{16}$, avaliaram as quedas apenas durante o período de intervenção. Hauer et al. ${ }^{10}$ e Morgan et al. ${ }^{13}$ avaliaram as quedas durante e após um período de intervenção, porém agruparam os dois períodos ao analisar os dados, o que não permite verificar o efeito do programa de exercícios e seu efeito prolongado após o término do mesmo.

Dos 10 estudos analisados, apenas dois ${ }^{10,16}$ compararam o risco relativo (RR) de quedas dos grupos que participaram de exercício físico com aqueles que receberam outros tipos de intervenção e $\mathrm{um}^{9}$ comparou o risco relativo de incidência (IRR) do período de seguimento após a intervenção com o período prospectivo avaliado antes da intervenção. Rubenstein et al. $^{7}$, que apesar de não terem realizado um acompanhamento após a intervenção, avaliaram o nível de atividade física e a taxa de quedas e compararam com o grupo controle, estabelecendo uma taxa de quedas por horas de atividade física realizada.

Os programas de exercícios propostos no estudo com o objetivo de reduzir ou prevenir o risco de quedas foram variados em sua duração, frequência, intensidade e modo de exercício. No quadro 2, é apresentada uma síntese dos artigos que relaciona os desenhos experimentais e os principais resultados. Cabe ressaltar que para o objetivo desta revisão o desfecho analisado em cada estudo foi exclusivamente em relação à ocorrência de quedas durante e após o término da intervenção e no período acompanhamento. 
Quadro 2. Síntese dos artigos que relaciona os desenhos experimentais de estudos sobre quedas em idosos.

\begin{tabular}{|c|c|c|c|c|c|}
\hline $\begin{array}{c}\text { Autores / } \\
\text { Ano / } \\
\text { País }\end{array}$ & $\begin{array}{l}\text { Amostra }(\mathrm{N}) / \\
\text { Idade Média } \\
\text { (anos) / } \\
\% \text { Feminino }\end{array}$ & $\begin{array}{l}\text { Tipo de estudo / } \\
\text { Seguimento } \\
\text { (pós-intervenção) }\end{array}$ & Variáveis & Intervenção & Resultados / Quedas \\
\hline $\begin{array}{l}\text { Rubenstein } \\
\text { et al. } 7 \\
\text { EUA }\end{array}$ & $\begin{array}{c}N=59 \\
G E=31 \\
\text { e } G C=28 / \\
74 \text { anos / } \\
59 \text { Homens }\end{array}$ & $\begin{array}{c}\text { RCT / } \\
\text { Não houve }\end{array}$ & $\begin{array}{c}\text { Força (MMII), } \\
\text { Funcionalidade, } \\
\text { QV, NAF } \\
\text { Nㅡㅁ quedas durante } \\
\underline{\text { a intervenção }}\end{array}$ & $\begin{array}{c}\text { GE: } 3 \times \text { sem / } 90 \text { min / } \\
12 \mathrm{sem} \\
\text { Força (MMII) + Exercícios } \\
\text { Aeróbicos + Exercícios de } \\
\text { equilíbrio. } \\
\text { GC: nada }\end{array}$ & $\begin{array}{c}\text { GE: } 6 \text { quedas/ } \\
1000 \text { horas } \\
x \\
\text { GC } 16,2 \text { quedas/ } \\
1000 \text { horas } \\
\nabla \text { de quedas ajustado } \\
\text { pelo NAF no GE } \\
(p \leq 0,05)\end{array}$ \\
\hline $\begin{array}{l}\text { Hauer et } \\
\text { al. } 10 \\
\text { Alemanha }\end{array}$ & $\begin{array}{c}N=57 \\
G E=31 \\
\text { e } G C=26 / \\
82 \text { anos / } \\
57 \text { Mulheres }\end{array}$ & $\begin{array}{c}\text { RCT / } \\
03 \text { meses }\end{array}$ & 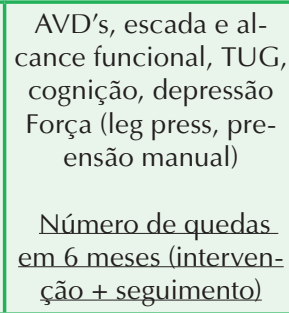 & $\begin{array}{c}\text { GE: } 3 \times \text { sem / } 12 \text { sem } \\
\text { Força (MMII) 2-3 séries / } \\
10-15 \text { rep. } 70-90 \% 1 \text { RM } \\
\text { Equilíbrio (dança e Tai Chi: } \\
45 \text { min) } \\
\text { GC: atividades placebo } \\
3 \times \text { sem / } 60 \mathrm{~min} / 12 \mathrm{sem}\end{array}$ & $\begin{array}{l}\text { Incidência de quedas: } \\
\text { GC: } 60 \% \text { x GE: } 45 \% \\
\text { v de } 25 \% \text { na incidên- } \\
\text { cia de quedas para o } \\
\text { GE (RR 0,753; CI } 95 \% \\
: 0,455-1,245) \text { (NS) }\end{array}$ \\
\hline $\begin{array}{l}\text { Steadman } \\
\text { et al.12 } \\
\text { Reino } \\
\text { Unido }\end{array}$ & $\begin{array}{c}\mathrm{N}=198 \\
\text { Ter Conv }=102 \\
\text { Ter } \\
\text { Conv+Equilíbrio } \\
=96 / \\
82 \text { anos } / \\
80 \% \text { mulheres }\end{array}$ & $\begin{array}{c}\mathrm{RCT} / \\
18 \text { semanas }\end{array}$ & $\begin{array}{c}\begin{array}{c}\text { Equilíbrio, velocidade } \\
\text { da marcha }\end{array} \\
\text { Número de quedas } \\
\text { no mês anterior (antes } \\
\text { e depois da interven- } \\
\text { ção, } 6 \text { e } 18 \text { semanas } \\
\text { após a intervenção) }\end{array}$ & $\begin{array}{c}\text { Ter Conv + Equilíbrio: } \\
2 \times \text { sem / } 45 \text { min / } 6 \text { sem } \\
\text { Alcance lateral; posição } \\
\text { tandem; pegar objeto do } \\
\text { chão, giros } \\
\\
\text { Ter Conv: } 2 \text { x sem / } 4 \text { sem } \\
\text { caminhada assistida, esca- } \\
\text { da e mobilidade }\end{array}$ & $\begin{array}{c}\boldsymbol{\nabla} \text { significativa } \\
(p=0,0001) \text { de } \\
\text { quedas a partir da } 6^{-} \\
\text {semana sendo susten- } \\
\text { tada até a 24 } 4^{\mathrm{a}} \text { semana } \\
\text { de estudo (18 sema- } \\
\text { nas pós-intervenção) } \\
\text { para os dois grupos } \\
\text { sem diferenças entre } \\
\text { os grupos. }\end{array}$ \\
\hline $\begin{array}{l}\text { Ballard et } \\
\text { al.15 } \\
\text { EUA }\end{array}$ & $\begin{array}{c}\mathrm{N}=40 \\
\mathrm{GE}=20 \\
(72,4 \text { anos }) \\
\mathrm{GC}=20 \\
\text { (73,4 anos) } \\
\text { 40 Mulheres }\end{array}$ & $\begin{array}{c}\text { RCT / } \\
12 \text { meses }\end{array}$ & $\begin{array}{l}\begin{array}{c}\text { Equilíbrio, agilidade, } \\
\text { alcance Funcional }\end{array} \\
\text { Número de quedas } \\
\frac{\text { durante a intervenção }}{(6 X) \text { e } 1 \text { ano após }} \\
\text { o término } \\
\text { da intervenção }\end{array}$ & $\begin{array}{l}\text { GE: } 3 \times \text { sem / } 60 \mathrm{~min} / \\
15 \mathrm{sem} \\
\text { Exercício aeróbio de baixo } \\
\text { impacto, força MMII e } \\
\text { MMSS e equilíbrio. } \\
\text { GC: nada }\end{array}$ & $\begin{array}{c}\text { Quedas (intervenção) } \\
\text { GE: } 4 \text { x GC: } 4 \text { (NS) } \\
\begin{array}{c}\text { Quedas (Seguimento } \\
\frac{1 \text { ano) }}{\text { GE: } 0 \text { x GC: } 6} \\
(p=0,106)(N S)\end{array}\end{array}$ \\
\hline $\begin{array}{l}\text { Morgan et } \\
\text { al.13 } \\
\text { EUA }\end{array}$ & $\begin{array}{c}\mathrm{N}=229 \\
\mathrm{GE}=119 \\
\mathrm{e} \mathrm{GC}=110 / \\
80,55 \text { anos / } \\
70,7 \% \\
\text { mulheres }\end{array}$ & $\begin{array}{c}\text { RCT / } \\
10 \text { meses }\end{array}$ & $\begin{array}{l}\text { Marcha, equilíbrio, } \\
\text { funcionalidade. } \\
\frac{\text { Número de quedas }}{\text { durante } 12 \text { meses }} \\
\frac{\text { (intervenção + segui- }}{\text { mento) }}\end{array}$ & $\begin{array}{c}\text { GE: } 3 \text { x sem / } 45 \text { min / } 8 \\
\text { sem } \\
\text { Força, equilíbrio, } \\
\text { marcha e flexibilidade } \\
\text { (fisioterapia) } \\
\text { GC: nada }\end{array}$ & $\begin{array}{c}\text { Quedas GE: } 28,6 \% \\
\text { x GC } 30,9 \% \\
\text { Risco de quedas: } \\
\text { v para GE com baixa } \\
\text { capacidade física (CF) } \\
\text { ( } p \leq 0,03, \text { HR: } 0,51) \\
\text { e } \Delta \text { para o grupo } \\
\text { alta CF } \\
\text { ( } p \leq 0,02 \text { HR: } 3,51) .\end{array}$ \\
\hline $\begin{array}{c}\text { Nitz \& } \\
\text { Choy14 } \\
\text { Austrália }\end{array}$ & $\begin{array}{c}N=73 \\
\text { GE (Worksta- } \\
\text { tion) }=37 \\
\text { e GC }=36 / \\
75,8 \text { anos / } \\
91,8 \% \\
\text { mulheres }\end{array}$ & $\begin{array}{c}\text { RCT / } \\
03 \text { meses }\end{array}$ & 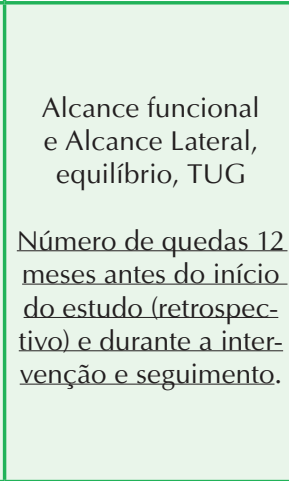 & $\begin{array}{c}\text { GE (Workstation) } \\
1 \text { x sem / } 60 \text { min / } 10 \text { sem } \\
\text { sentar e levantar, passo a } \\
\text { frente e atrás; alcance e } \\
\text { jogos c/ bola } \\
\text { GC: } 1 \text { x sem / } 40 \text { min / } \\
10 \text { sem } \\
\begin{array}{c}\text { Exercícios similares ao GE } \\
\text { de intensidade mais leves }\end{array}\end{array}$ & $\begin{array}{c}\text { Quedas } 12 \text { meses } \\
\text { antes: } \\
G E=41 \mathrm{E} \mathrm{GC}=44 \\
\text { Quedas (intervenção): } \\
\text { GE }=12 \text { e GC }=13 \\
\text { Quedas (seguimento } \\
\underline{3 \text { meses): }} \\
\mathrm{GE}=9 \text { e GC }=9 \\
\mathrm{GE} \text { mostrou uma } \\
\text { maior } \mathbf{\nabla} \text { às quedas } \\
\text { ( } p=0,000) \text { e GC } \\
\text { também ( } p=0,024) \\
\text { sem diferença entre } \\
\text { os grupos }\end{array}$ \\
\hline
\end{tabular}




\begin{tabular}{|c|c|c|c|c|c|}
\hline $\begin{array}{l}\text { Autores / } \\
\text { Ano / } \\
\text { País }\end{array}$ & $\begin{array}{l}\text { Amostra }(\mathrm{N}) / \\
\text { Idade Média } \\
\text { (anos) / } \\
\% \text { Feminino }\end{array}$ & $\begin{array}{l}\text { Tipo de estudo / } \\
\text { Seguimento } \\
\text { (pós-intervenção) }\end{array}$ & Variáveis & Intervenção & Resultados / Quedas \\
\hline $\begin{array}{c}\text { Li et al.11 } \\
\text { EUA }\end{array}$ & $\begin{array}{l}\mathrm{N}=256 \\
\text { GTC (Tai Chi) } \\
=125 \\
\text { GC (alonga- } \\
\text { mentos) }=131 \\
77,48 \text { anos / } \\
70 \% \text { mulhe- } \\
\text { res / }\end{array}$ & $\begin{array}{l}\mathrm{RCT} / \\
6 \text { meses }\end{array}$ & $\begin{array}{l}\text { Equilíbrio, marcha, } \\
\text { alcance funcional. } \\
\text { Número de quedas } \\
\text { durante a intervenção } \\
\text { e durante o segui- } \\
\text { mento (pós-interven- } \\
\text { ção) }\end{array}$ & $\begin{array}{c}\text { GTC: } 3 \times \text { sem / } 60 \text { min / } \\
26 \text { sem } \\
\text { GC: } 3 \times \text { sem / } 60 \text { min / } 26 \\
\text { sem (alongamentos apenas } \\
\text { para parte superior do } \\
\text { corpo) }\end{array}$ & 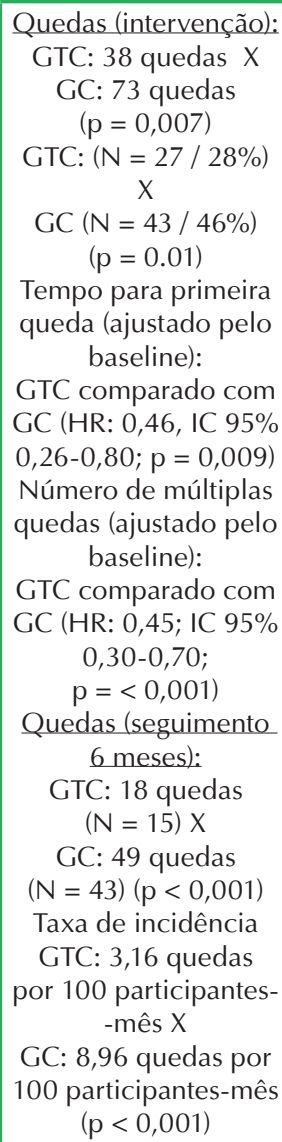 \\
\hline $\begin{array}{c}\text { Means et } \\
\text { al.8 } \\
\text { EUA }\end{array}$ & $\begin{array}{c}\mathrm{N}=338 \\
\mathrm{GE}=181 \text { e GC } \\
=157 / \\
73,5 \text { anos } / \\
57 \% \text { mulheres }\end{array}$ & $\begin{array}{c}\text { RCT / } \\
06 \text { meses }\end{array}$ & $\begin{array}{c}12 \text { tarefas orientadas } \\
\text { envolvendo tarefas } \\
\text { realizadas em casa; } \\
\text { AVD's, mobilidade e } \\
\text { força. } \\
\text { Número de quedas } \\
\text { relatadas } 6 \text { meses } \\
\text { antes da intervenção } \\
\text { e durante os } 6 \text { meses } \\
\text { de seguimento (pós- } \\
\text {-intervenção) }\end{array}$ & $\begin{array}{l}3 \times \text { sem / } 90 \text { min / } 6 \text { sem } \\
\text { alongamento, controle } \\
\text { postural, caminhada, coor- } \\
\text { denação, força, Intensida- } \\
\text { de (13 - Borg) } \\
\text { GC: seminários de interes- } \\
\text { se geral (não relacionados } \\
\text { a quedas) }\end{array}$ & $\begin{array}{c}\text { Quedas } 6 \text { meses } \\
\text { antes: } \\
\text { GE: } 2,01 \text { e GC: } 0,48 \\
\text { quedas / GE: } 1,05 \text { e } \\
\text { GC: } 0,31 \text { lesões rela- } \\
\text { cionadas a quedas } \\
\text { Quedas (seguimento } \\
\underline{6 \text { meses): }} \\
\text { GE: } 0,24 \text { e GC: } 0,59 \\
\text { quedas / GE: } 0,18 \text { e } \\
\text { GC: } 0,52 \text { lesões rela- } \\
\text { cionadas a quedas. }\end{array}$ \\
\hline $\begin{array}{l}\text { Weerdes- } \\
\text { teyn et al.9 } \\
\text { Holanda }\end{array}$ & $\begin{array}{c}\mathrm{N}=107 \\
\mathrm{GE}=79 \text { e GC } \\
=28 / \\
74,2 \text { anos } / \\
73,5 \% \text { mu- } \\
\text { Iheres }\end{array}$ & $\begin{array}{c}\text { RCT / } \\
6 \text { meses }\end{array}$ & $\begin{array}{l}\text { Equilíbrio, Tempo de } \\
\text { reação, Coordenação. } \\
\frac{\text { Número de quedas }}{\text { relatadas e super- }} \\
\frac{\text { visionadas } 6 \text { meses }}{\text { antes da interven- }} \\
\text { ção (Prospectivo) e } \\
\frac{\text { durante o seguimento }}{\text { (pós-intervenção) }}\end{array}$ & $\begin{array}{l}\text { GE: } 2 \text { X sem / } 90 \text { min / } \\
5 \text { sem } \\
\text { Exercícios de equilíbrio, } \\
\text { marcha e coordenação } \\
\text { que imitavam as AVD's, } \\
\text { caminhadas em ambien- } \\
\text { tes diferentes. Estratégias } \\
\text { de prevenção de que- } \\
\text { das (reconhecimento e } \\
\text { enfrentamento de fatores } \\
\text { potencialmente perigo- } \\
\text { sos e técnicas de caídas } \\
\text { realizadas) } \\
\text { GC: nada }\end{array}$ & $\begin{array}{c}\boldsymbol{\nabla} \text { de } 46 \% \text { nas quedas } \\
\text { no GE comparado ao } \\
\text { período do baseline } \\
\text { (incidence rate ratio } \\
\text { [IRR]: } 0,54, \text { IC } 95 \% \\
0,36-0,79 \text { ) e } \boldsymbol{\nabla} \text { de } \\
46 \% \text { (IRR: 0,54, IC } \\
\text { 95\% 0,34-0,86) com- } \\
\text { parado ao GC. Para } \\
\text { o GC a } \boldsymbol{\nabla} \text { de quedas } \\
\text { foi (IRR: } 0,98, \text { IC } 95 \% \\
0,56-1,72 \text { ) comparado } \\
\text { ao periodo baseline } \\
\text { A proporção de } \\
\text { caidores no GE } \boldsymbol{\nabla} \text { de } \\
58 \% \text { para } 40 \% \text { (IRR } \\
0,61,0,38-0,98 \text {.) A } \\
\text { proporção de caido- } \\
\text { res no GC (32\%) } \\
\text { não se modificou } \\
\text { (IRR: 0,71, iC 95\% } \\
0,28-1,78 \text { ) }\end{array}$ \\
\hline
\end{tabular}




\begin{tabular}{|c|c|c|c|c|c|}
\hline $\begin{array}{c}\text { Autores / } \\
\text { Ano / } \\
\text { País }\end{array}$ & $\begin{array}{l}\text { Amostra }(\mathrm{N}) / \\
\text { Idade Média } \\
\text { (anos) / } \\
\% \text { Feminino }\end{array}$ & $\begin{array}{l}\text { Tipo de estudo / } \\
\text { Seguimento } \\
\text { (pós-intervenção) }\end{array}$ & Variáveis & Intervenção & Resultados / Quedas \\
\hline $\begin{array}{l}\text { Shumway- } \\
\text {-Cook et } \\
\text { al.16 } \\
\text { EUA }\end{array}$ & $\begin{array}{l}N=453 \\
G E=226 \text { e GC } \\
=227 / \\
75,6 \text { anos / } \\
77 \% \text { mulheres }\end{array}$ & $\begin{array}{l}\text { RCT / } \\
\text { Não Houve }\end{array}$ & $\begin{array}{l}\text { Estado mental; histó- } \\
\text { rico de saúde; teste } \\
\text { de sentar e levantar; } \\
\text { equilíbrio de Berg; } \\
\text { TUG } \\
\text { Número de quedas } \\
\underline{\text { durante a intervenção }}\end{array}$ & $\begin{array}{l}3 \times \text { sem / } 60 \text { min / } \\
12 \text { meses } \\
30 \text { min aeróbio; } 20 \text { min } \\
\text { treino progressivo de } \\
\text { força; } 10 \text { min flexibilidade } \\
\text { e equilíbrio } \\
\text { GC: } 2 \text { manuais para } \\
\text { prevenção }\end{array}$ & $\begin{array}{l}\text { A taxa de incidência } \\
\text { de quedas foi } 25 \% \\
\nabla \text { no GE comparado } \\
\text { com GC }(1,33 \text { vs } 1,77 \\
\text { quedas/pessoas por } \\
\text { ano (RR }=0,75 \text {, IC } 95 \\
\% 0,52-1,09) \text { (NS) }\end{array}$ \\
\hline
\end{tabular}

GE: Grupo Experimental / GC: Grupo Controle / RCT: Randomized Controlled Trial / MCT: Multi-centre Controlled Trial / CBCT: Community Based Controlled Trial / NR: Não Relatado / NS: Não Significativo / MMII: Membros inferiores / QV: Qualidade de vida / NAF: Nível de Atividade Física / AVD's: Atividades da Vida Diária / TUG: Time up and go / Ter Conv: terapia convencional / 1 RM: Teste de 1 Repetição Máxima / RR: Rate Ratio / HR: Hazard Ratio / IRR: Incidence Rate Ratio

\section{DISCUSSÃO}

O principal objetivo deste estudo foi analisar, mediante revisão sitemática da literatura, se programas de exercícios físicos planejados, orientados e regulares podem reduzir a ocorrência de quedas acidentais em idosos. Outras questões associadas dizem respeito aos componentes do programa, ou seja, a modalidade, frequência, duração e intensidade. Dos 10 estudos analisados nesta revisão, sete apresentaram resultados significativos na redução de quedas em idosos ${ }^{7-9,11-14}$.

Estes estudos tiveram em comum a inclusão de exercícios de força, equilíbrio e tarefas de funcionalidade relacionadas às atividades da vida diária. A inclusão destes exercícios na prevenção e redução de quedas justifica-se, pois a redução da força muscular, alterações de equilíbrio, modificações no padrão da marcha são fatores de risco para quedas em idosos e afetam a funcionalidade do idoso ${ }^{1-4}$.

No entanto, houve diferença quanto ao número de sessões semanais e à duração do programa, o que dificulta a análise. Tais diferenças limitam a possibilidade de estabelecer diretrizes seguras para estabelecer protocolos e procedimentos que possam efetivamente prevenir e reduzir quedas. A intensidade do exercício não foi objetivamente determinada na maioria dos estudos, dificultando a reprodução dos procedimentos experimentais por outros pesquisadores e a orientação prática para professores e terapeutas.

O período de acompanhamento é outra variável importante que pode confundir a análise dos resultados. Nitz e Choy ${ }^{14}$, por exemplo, compararam o número de quedas ocorrido no ano anterior à pesquisa com a ocorrência de quedas nos três meses após o tratamento. Esta diferença pode ter subestimado a ocorrência de quedas, além da desistência dos participantes que foi elevada durante o estudo, principalmente, durante o período de acompanhamento. No estudo de Steadman et al. ${ }^{12}$, o tempo de seguimento foi de 18 semanas, porém os autores compararam os resultados com o número de quedas ocorridas no mês anterior ao início do estudo. A maioria dos estudos retrospectivos analisa a ocorrência de quedas, pelo menos, nos seis meses que precedem o início da pesquisa.

Rubenstein et al. ${ }^{7}$ não realizaram acompanhamento após o programa de exercícios e avaliaram as quedas ocorridas durante o período de treinamento e estabeleceram uma taxa de quedas para cada 1000 horas de exercício, atividades físicas ou de lazer. Os autores argumentaram que os idosos que participaram do programa de exercício, saíam mais de casa e estavam mais expostos aos riscos proporcionados pelo ambiente e, mesmo assim, tiveram menor taxa de quedas do que os sujeitos do grupo controle. Os próprios autores sugerem que para melhor análise dos efeitos do exercício é necessário realizar um acompanhamento mais longo (pelo menos um ano).

As diferenças entre frequência semanal e a duração total dos programas que apresentaram redução da ocorrência de quedas sugere que a resposta ao exercício pode estar mais relacionada às características iniciais dos participantes. No estudo de Nitz \& $\mathrm{Ch}{ }^{14}$, por exemplo, além do período curto de seguimento já citado, foi realizada apenas uma sessão semanal de exercícios durante 10 semanas, os participantes tinham idade média 75,8 anos e na maioria baixo nível de atividade física.

Steadman et al..$^{12}$, prescreveram duas sessões semanais por seis semanas, para dois grupos, um que realizava terapia convencional (fisioterapia) com outro grupo que associou exercícios de equilíbrio ao tratamento, sem grupo controle. Porém a amostra era composta por idosos fragilizados, com média de idade de 82 anos e com problemas diagnosticados 
de equilíbrio. Isto pode explicar porque um período de tratamento tão curto possa ter apresentado resultados positivos, embora se deva destacar que os benefícios foram mantidos por até 24 semanas. Resultados favoráveis foram encontrados também por Morgan et al. ${ }^{13}$, que avaliaram idosos com baixa e alta aptidão física e houve redução do número de quedas apenas nos participantes que apresentaram, no início do programa, uma baixa capacidade física.

Means et al. ${ }^{8}$ avaliaram os efeitos de uma intervenção de curto prazo (seis semanas), no equilíbrio, mobilidade e número de quedas em idosos com média de idade de 73 anos (57\% mulheres). Compararam o número de quedas durante seis meses após a intervenção com as quedas ocorridas 6 meses antes do programa. O programa foi composto por exercícios de força com uso de elásticos em intensidade moderada (percepção subjetiva de esforço) e tarefas do dia-a-dia. Neste estudo, o grupo de exercício e de controle foi composto por idosos com e sem histórico de quedas, no entanto, o grupo experimental apresentou uma maior taxa de quedas que o controle, antes do início do programa, o que pode sugerir que o grupo experimental foi composto por idosos com maior comprometimento físico.

Os resultados do estudo realizado por Weerdesteyn et al. ${ }^{9}$, com apenas cinco semanas de intervenção (10 sessões), apresentou redução de 46\% do número de quedas quando comparado aos dados iniciais e ao grupo controle. Tais melhorias podem ser atribuídas à especificidade do programa. Os sujeitos realizavam atividades em um circuito com as características semelhantes aos desafios encontrados pelos idosos ao se deslocar, com pisos irregulares e obstáculos.

Três estudos desta revisão não apresentaram resultados significativos em relação à redução do número de quedas. Hauer et al. ${ }^{10}$ observaram uma redução de $25 \%$ na incidência de quedas, que não foram significativos em comparação ao grupo controle. No entanto, neste estudo o grupo controle realizou atividade física (placebo) envolvendo flexibilidade, exercícios calistênicos e jogos com bola, que podem ter influenciado os resultados do controle. Na pesquisa de Ballard et al. ${ }^{15}$, os dados do seguimento foram reportados apenas um ano após o final da intervenção, o que pode ter gerado imprecisão nas respostas. Shummay et $a .^{16}$, não encontraram diferenças, no entanto, a seleção dos participantes pode ter sido um fator de confusão, pois $73 \%$ dos participantes não tinham histórico de quedas e 50\% não apresentavam fatores de risco.

\section{CONCLUSÃO}

Os resultados sugerem que o exercício físico, de forma isolada, é capaz de reduzir o risco de quedas, isto ficou evidente em sete dos estudos avaliados. Exercícios de equilíbrio e força muscular são as estratégias mais presentes nas intervenções propostas, no entanto, uma indicação clara em relação aos principais componentes do exercício (frequência; duração; intensidade) não foi encontrada.

As características da população parecem ter influência nos resultados, a idade e a condição física podem determinar o grau de adaptação obtida com o programa de exercício mesmo quando a frequência, intensidade e duração dos programas diferem. O período de observação de ocorrência de quedas antes do início da intervenção, o tempo de duração do acompanhamento pós-exercício devem ser considerados, períodos curtos podem subestimar a ocorrência de quedas. Em resumo, parece que estudos que associaram componentes de força e/ou equilíbrio, além de outras formas de intervenção, que tenham sido realizados, no mínimo, duas vezes por semana e que tenham acompanhado os indivíduos, em média, de 3 a 6 meses após a intervenção, mostraram-se mais efetivos em reduzir e prevenir as quedas em idosos vivendo na comunidade.

Para conclusões mais definitivas é necessário que o planejamento do programa de exercícios com objetivo de reduzir quedas, utilize métodos adequados para identificar as pessoas mais suscetíveis a sua ocorrência, utilizar formas confiáveis para investigar a sua ocorrência e acompanhar os sujeitos em períodos de tempo curtos (mensal) para obter informações mais precisas. Quanto ao programa de exercícios, sugere-se maior detalhamento sobre sua composição, com a finalidade de permitir a sua reprodução por outros pesquisadores. Outra forma para analisar o risco de quedas pode ser a exposição controlada de situações experimentais que induzam os idosos a situações de distúrbio similares às condições reais de queda ${ }^{17}$.

\section{REFERÊNCIAS BIBLIOGRÁFICAS}

1. Hill K, Schwarz J. Assessment and management of falls in older people. Intern Med J 2004;34(9-10):557-64.

2. Guideline for the prevention of falls in older persons. American Geriatrics Society, British Geriatrics Society, and American Academy of Orthopaedic Surgeons Panel on Falls Prevention. J Am Geriatr Soc 2001;49(5):664-72. 
3. Perracini MR, Ramos LR. Fatores associados a quedas em uma coorte de idosos residentes na comunidade. Rev Saude Publica 2002;36(6):709-16.

4. Masud T, Morris RO. Epidemiology of falls. Age Ageing 2001;30(Suppl4):3-7.

5. Bento PC, Pereira G, Ugrinowitsch C, Rodacki AL. Peak torque and rate of torque development in elderly with and without fall history. Clin Biomech (Bristol, Avon) 2010;25(5):450-4.

6. Moylan KC, Binder EF. Falls in older adults: risk assessment, management and prevention. Am J Med 2007;120(6):493.e1-6.

7. Rubenstein LZ, Josephson KR, Trueblood PR, Loy S, Harker JO, Pietruskazka FM, Robbins AS. Effects of a group exercise program on strength, mobility, and falls among fall-prone elderly men. J Gerontol A Biol Sci Med Sci 2000;55(6):M317-21

8. Means KM, Rodell DE, O'Sullivan PS. Balance, mobility, and falls among community-dwelling elderly persons: effects of a rehabilitation exercise program. Am J Phys Med Rehabil 2005;84(4):238-50.

9. Weerdesteyn V, Rijken H, Geurts AC, Smits-Engelsman BC, Mulder T, Duysens J. A five-week exercise program can reduce falls and improve obstacle avoidance in the elderly. Gerontology 2006;52(3):131-41.

10. Hauer K, Rost B, Rutschle K, Opitz H, Specht N, Bartsch P, Oster P, Schlierf G. Exercise training for rehabilitation and secondary prevention of falls in geriatric patients with a history of injurious falls. J Am Geriatr Soc 2001;49(1):10-20.

11. Li F, Harmer PK, Fisher KJ, McAuley E, Chaumeton N, Eckstrom E, Wilson NL. Tai Chi and fall reductions in older adults: a randomized controlled trial. Journal Gerontol A Biol Sci Med Sci 2005;60(2):187-94.

12. Steadman J, Donaldson N, Kalra L. A randomized controlled trial of an enhanced balance training program to improve mobility and reduce falls in elderly patients. J Am Geriatr Soc 2003;51(6):847-52.
13. Morgan RO, Beth A, Virnig BA, Duque M, Abdel-Moty E, DeVito CA. Low-Intensity exercise and reduction of the risk for falls among at-risk elders. J Gerontol A Biol Sci Med Sci 2004;59(10):1062-7.

14. Nitz JC, Choy NL. The efficacy of a specific balance-strategy training program for preventing falls among older people: a pilot randomised controlled trial. Age Ageing 2004:33(1):52-8.

15. Ballard JE, McFarland C, Wallace LS, Holiday DB, Roberson G. The effect of 15 weeks of exercise on balance, leg strength, and reduction in falls in 40 women aged 65 to 89 years. J Am Med Womens Assoc 2004;59(4):255-61

16. Shumway-Cook A, Silver IF, LeMier M, York S, Cummings P, Koepsell TD. Effectiveness of a community-based multifactorial intervention on falls and fall risk factors in community-living older adults: a randomized, controlled trial. J Gerontol A Biol Sci Med Sci 2007;62(12):1420-7.

17. Pijnappels, M., van der Burg, P. J., Reeves, N. D., van Dieen, J. H., Identification of elderly fallers by muscle strength measures. Eur J Appl Physiol 2008;102:585-92.

\author{
Endereço para correspondência \\ Paulo Cesar Barauce Bento \\ Universidade Federal do Paraná \\ Setor de Ciências Biológicas. Departamento \\ de Educação Física \\ R. Coração de Maria, 92 - BR 116 km 95 \\ Jardim Botânico \\ CEP 80215-370 - Curitiba, PR. Brasil. \\ E-Mail: p.bento@yahoo.com.br
}

